\title{
Evaluación preclínica y estudio de estabilidad de extractos a partir del follaje de Momordica charantia Lin
}

\author{
LAGARTO, A.; SÁNCHEZ, E.; PILOTO, J.; REMIGIO, A.; BARZAGA, P.; RODRÍGUEZ, C.; CARBALLO, C.; \\ COURET, M.; VEGA, Y.; LÓPEZ, R. \\ Centro de Investigación y Desarrollo de Medicamentos. CIDEM. Ave 26 y Calzada de Puentes Grandes, $N^{\circ}$ \\ 1605, Plaza, Ciudad Habana, Cuab, CP 10600. Correspondencia: Alicia Lagarto. Lab. Control Biológico.17 \\ No. 6208 e/ 62 y 64, Playa, Ciudad Habana, Cuba. Teléfono: 209 5451. E-mail: alicia.lagarto@cidem.sld.cu y \\ alicialp@infomed.sld.cu.
}

\begin{abstract}
RESUMEN: El objetivo del presente trabajo fue la evaluación preclínica y el estudio de estabilidad de extractos a partir del follaje de Momordica charantia Lin. Se obtuvieron extractos acuoso e hidroalcohólico para los cuales se establecieron las especificaciones de calidad mediante la evaluación de tres lotes y se estudió su estabilidad por el método de vida de estante durante 12 meses. A los extractos se le evaluó el potencial genotóxico mediante ensayos de micronúcleos en médula ósea de ratón y aberraciones cromosómicas en linfocitos de sangre periférica. La actividad hipoglicemiante oral fue evaluada en animales con hiperglicemia temporal inducida por carga de glucosa. Como resultados se establecieron las especificaciones de calidad de los extractos acuoso e hidroalcohólico, los mismos mostraron estabilidad por 6 meses para el extracto acuoso y 12 meses para el extracto hidroalcohólico. No mostraron efecto genotóxico en los ensayos evaluados y mostraron efecto hipoglicemiante oral a la dosis de $450 \mathrm{mg} / \mathrm{kg}$.
\end{abstract}

Palabras clave: Momordica charantia Lin, extractos, estabilidad, genotoxicidad, hipoglicemiante.

\begin{abstract}
Obtaining and characterizing the Momordica charantia Linn leaf extracts. The objective of this investigation was the preclinical evaluation and the stability study of the Momordica charantia Linn hydroalcoholic and aqueous leaf extracts. The hydroalcoholic and aqueous extracts were obtained and the quality specifications were determined by evaluating three lots. The stability of the extracts was evaluated for 12 months. The genotoxic potential of the extracts was evaluated by mouse bone marrow micronucleus test and chromosome aberration test. The hypoglycemic effect was determined by oral glucose tolerance test. As results, the quality specifications were established and the aqueous extract was stable for 6 months and the hydroalcoholic extract for 12 months. A genotoxic effect was not observed in both extracts and the hypoglycemic effect was observed at the oral dose of $450 \mathrm{mg} / \mathrm{kg}$ of body weight.
\end{abstract}

Keywords: Momordica charantia Linn, extracts, genotoxicity, hypoglycemic effect.

\section{INTRODUCCIÓN}

La diabetes mellitus constituye un gran problema de salud que afecta actualmente más de 194 millones de personas en todo el mundo. Esta enfermedad se caracteriza por una hiperglicemia crónica, específicamente una hiperglicemia postpandrial estrechamente relacionada con complicaciones cardiovasculares y la muerte (Ceriello, 2005).

Momordica charantia Lin, conocida como "cundeamor", es un bejuco anual trepador perteneciente a la familia Cucurbitacea. La planta es muy común en las cercas de patios, fincas y potreros de toda la isla (Roig \& Mesa, 1974). En la farmacología y actividad biológica de $M$. charantia, varios autores han estudiado el efecto hipoglicémico de la pulpa del fruto, semillas y planta entera, reportándose actividad de la planta para disminuir los niveles de glucosa (Miura et al. 2004).

El extracto metanólico provoca disminución de niveles de glucosa, triglicéridos y lipoproteínas de baja densidad, así como un incremento de lipoproteínas de alta densidad (Chaturvedi et al. 2004), pudiendo ser efectivo para el tratamiento de la hiperlipidemia además de hipoglicemiante (Ahmed et al. 2004).

Por otro lado, $M$. charantia también reduce 
los niveles de insulina y glucosa sérica en ratas con obesidad inducida y ratones con diabetes tipo 2 , aunque no posee efectos en ratones normales (Miura et al. 2004; Miura et al. 2001; Chen et al. 2003). Estos datos sugieren que esta planta contiene varios componentes que disminuyen la hiperglicemia y la incidencia de diabetes mellitus.

Los extractos de $M$. charantia poseen potencial terapéutico avalado científicamente que posibilitan el empleo de esta planta en diversas patologías, especialmente en la diabetes, por lo que el estudio de esta especie en nuestro país es de gran importancia para avalar el empleo de esta planta como agente terapéutico. El presente trabajo tiene como objetivo la evaluación preclínica y el estudio de estabilidad de extractos a partir de M. charantia que pretendemos emplear para la obtención de productos terminados destinados al tratamiento de la diabetes.

\section{MATERIALES Y MÉTODOS}

\section{Sustancias de prueba}

Los extractos fueron obtenidos empleando el follaje de $M$. charantia, la cual fue cultivada y cosechada en la Estación Experimental de Plantas Medicinales “Juan Tomás Roig”, en Güira de Melena, Artemisa, Cuba. El espécimen de dicha planta fue conservado con $\mathrm{N}^{\circ}$ de herbario 4778 . El material vegetal fue lavado y desinfectado empleando solución de hipoclorito de sodio, secado en estufa a $40^{\circ} \mathrm{C}$ y molido para la obtención de los extractos.

\section{Animales}

Todos los animales empleados en los ensayos fueron suministrados por el Centro para la Producción de Animales de Laboratorio (CENPALAB). Los mismos se mantuvieron en una sala con temperatura controlada de $22 \pm 2^{\circ} \mathrm{C}$ y ciclo luz - oscuridad de 12/12 horas. La alimentación consistió en dieta standard para ratas y ratones proveniente del CENPALAB y agua acidulada a voluntad. Todos los ensayos fueron realizados de acuerdo a las Buenas Prácticas de Laboratorio.

\section{Obtención de extractos}

El extracto acuoso se obtuvo por método de reflujo empleando agua como solvente en proporción 1:10 (droga/solvente), el mismo fue filtrado y preservado. El extracto hidroalcohólico fue obtenido por método de repercolación, según NRSP-311 (MINSAP 1992) empleando etanol 30\% como solvente en una proporción droga/solvente de $1: 1$.

\section{Estudio de estabilidad}

Para el estudio de estabilidad se elaboraron tres lotes de cada extracto, de 2 litros cada uno, los cuales fueron envasados en frascos de vidrio color ámbar de $120 \mathrm{ml}$ de capacidad con tapa de polipropileno, los cuales fueron almacenados a temperatura ambiente protegidos de la luz.

Se realizó el estudio de estabilidad de vida estante a la temperatura de $30 \pm 2{ }^{\circ} \mathrm{C}$ y humedad relativa de $70 \pm 5 \%$ por un período de un año. Los análisis fueron realizados al inicio, 3, 6, 9 y 12 meses. Los índices de calidad estudiados para la caracterización de los extractos se reflejan en la Tabla 1. Se emplearon los métodos analíticos según NRSP-309 (MINSAP 1992), NRSP-311 (MINSAP 1992) y Atlas de cromatografía en capa delgada (Wagner \& Bladt, 2001). Ambos extractos fueron

TABLA 1. Índices de calidad y especificaciones para los extractos de M. charantia.

\begin{tabular}{ccc}
\hline Ensayos & Extracto acuoso & Especificaciones \\
\cline { 2 - 3 } & $\begin{array}{c}\text { Liquido de color pardo claro, no } \\
\text { transparente, de olor característico }\end{array}$ & $\begin{array}{c}\text { Liquido de color pardo oscuro, olor característico, } \\
\text { nescripción }\end{array}$ \\
\hline PH & $6.0-8.0$ & $6.0-8.0$ \\
\hline Índice de refracción & $1.330-1.339$ & $1.360-1.375$ \\
\hline Sólidos totales & $>1.20 \%$ & $>5.0 \%$ \\
\hline Densidad relativa & $1.002-1.020$ & $1.000-1.060$ \\
\hline Contenido alcohólico & - & $>18.0 \%$ \\
\hline Identificación & Perfil cromatográfico de principios amargos & Perfil cromatográfico de principios amargos \\
\hline Conteo microbiano & CB $<10^{3}$ UFC & CB $<10^{3}$ UFC \\
& CH $<10^{2}$ UFC & CH $<10^{2}$ UFC
\end{tabular}


caracterizados por cromatografía de capa delgada empleando placas de silica gel F25, sistema de solvente de cloroformo - metanol 95:5 y revelado con ácido fosfórico - vainillina.

\section{Evaluación de la genotoxicidad}

\section{de ratón \\ Ensayo de micronúcleos en médula ósea}

Se emplearon 50 ratones Cenp: NMRI con edad entre 8 y 12 semanas y peso corporal de 23$25 \mathrm{~g}$. Se establecieron cinco grupos experimentales de 5 animales/sexo/grupo: control negativo (vehículo), control positivo (Ciclofosfamida, $40 \mathrm{mg} /$ $\mathrm{kg}$ ) y tres dosis del extracto $112.5,225$ y $450 \mathrm{mg} / \mathrm{kg}$, administradas en un volumen de $10 \mathrm{~mL} / \mathrm{kg}$ de peso corporal por vía intragástrica.

Se realizaron 2 administraciones consecutivas con intervalos de 24 horas y se procedió al sacrificio $24 \mathrm{~h}$ después de la última aplicación. Para la obtención de las preparaciones de la médula ósea se siguió un procedimiento descrito con anterioridad (Schmid, 1976), cuantificando el número de eritrocitos policromáticos (PCE) portadores de micronúcleos (PCE-MN) y expresándolo como porcentaje con respecto al total de PCE observados (2000 por animal). Como criterio de genotoxicidad se tuvo en cuenta la significación estadística del incremento del \% PCE-MN en una o más dosis con respecto al control negativo. Como criterio de toxicidad se tomó la significación estadística de la disminución de la proporción de eritrocitos policromáticos/normocromáticos (PCE/NCE) en una o más dosis con respecto al control negativo (Hayashi, 1994).

Las diferencias entre el grupo control negativo y los grupos tratados, fueron analizadas con los valores reales para ambos índices o después de adecuadas trasformaciones de escala al realizar las pruebas de Kolmogorov-Smirnov y Bartlett. Posteriormente se realizó un análisis de varianza (ANOVA) bifactorial.

\section{Ensayo de aberraciones cromosómicas}

El ensayo se llevó a cabo en linfocitos de sangre periférica, los cuales fueron cultivados en medio con fitohemaglutinina a $37^{\circ} \mathrm{C}$. El extracto hidroalcohólico concentrado fue evaluado a las concentraciones de 1,2 y $5 \mathrm{mg} / \mathrm{ml}$ en RPMI. Como controles positivos se emplearon Mitomicina-C $0.25 \mu \mathrm{g} / \mathrm{ml}$ (sin S9) y Ciclofosfamida $12 \mu \mathrm{g} / \mathrm{ml}$ (con S9), como control negativo se empleó medio RPMI 1640

Las células en crecimiento se trataron con la sustancia de ensayo en presencia y en ausencia de un sistema de activación metabólica (S9) 48 horas después de la estimulación mitogénica. Se prepararon dos cultivos para cada concentración. Los cultivos fueron tratados con colchicina entre 1-3 horas antes de la recolección, los mismos se recolectaron y procesaron por separado para preparar los cromosomas. Se analizaron 200 metafases bien extendidas en cada concentración y control y, en su caso, repartidas de forma equilibrada entre los cultivos dobles.

Para la interpretación de los resultados se siguieron los criterios de aumento estadísticamente significativos del número de células que producen aberraciones cromosómicas y relación dosisrespuesta (Tice, 1995; Hartmann et al. 2003).

\section{Evaluación farmacológica}

Se evaluó el efecto hipoglicemiante de los extractos en modelo de hiperglicemia temporal inducida por carga de glucosa, empleando ratas Wistar machos, de 150 a $200 \mathrm{~g}$ de peso. Se establecieron cinco grupos experimentales de 8 animales cada uno: control negativo (vehículo), control positivo (Glibenclamida $10 \mathrm{mg} / \mathrm{kg}$ ) y tres dosis del extracto 50,150 y $450 \mathrm{mg} / \mathrm{kg}$, administradas por vía intragástrica durante 7 días para el extracto hidroalcohólico y 21 días para el extracto acuoso.

Dieciocho horas antes de la última administración se retiraron los alimentos sólidos manteniéndose el acceso al agua ad libitum. Se realizó una extracción de sangre del plexo retroorbital, administrando seguidamente los tratamientos correspondientes. Una hora más tarde se realizó la administración de $3 \mathrm{~g} / \mathrm{kg}$ de una solución de D-glucosa (600 mg/ml). Posteriormente se realizaron extracciones de sangre teniendo en cuenta los siguientes intervalos de tiempo a partir de la administración de la glucosa: $30 \mathrm{~min}, 1 \mathrm{~h}$, $2 \mathrm{~h}, 3 \mathrm{~h}, 4 \mathrm{~h}$ y $6 \mathrm{~h}$ (CYTED 1995). Las muestras de sangre obtenidas fueron centrifugadas a $3000 \mathrm{rpm}$ por 15 minutos para obtener el suero. La glicemia se determinó usando el kit reactivo RapiGlucoTest, mediante un método enzimático y lectura en espectrofotómetro a $500 \mathrm{~nm}$. Los valores de glicemia se expresaron en mmol/l (CYTED 1995).

Los resultados fueron expresados como el valor medio \pm error estándar de la media. El procesamiento estadístico de los resultados se llevó a cabo empleando un ANOVA y la prueba de rangos múltiples de Duncan.

\section{RESULTADOS}

\section{Estudio de estabilidad}

Los índices de calidad evaluados para el extracto hidroalcohólico durante 12 meses se mantuvieron dentro de los límites establecidos por las especificaciones. Para el extracto acuoso 
el contenido de sólidos totales disminuyó significativamente $(p<0.01)$ a partir de los 9 meses al compararlo con los resultados obtenidos al inicio del estudio (Figura 1).

El estudio cromatográfico mostró para el extracto acuoso una banda solitaria en el intervalo de Rf $0.62-0.65$ de color pardo violáceo semejante a la del aglicon charantina, la cual se mantuvo presente hasta el final del experimento en los tres lotes. Para el extracto hidroalcohólico se observaron un total de 9 bandas de color pardo claro característico para la detección del glicosido cucurbitacina. Al final del experimento solo se observaron 5 de ellas que correspondían a las de mayor intensidad al inicio del mismo en los intervalos de Rf $0.18-0.22,0.33$ $-0.37,0.5-0.52$ y $0.57-0.61$.

\section{Evaluación de la genotoxicidad}

\section{de ratón \\ Ensayo de micronúcleos en médula ósea \\ Los resultados del ensayo aparecen en la}

Tabla 2. En ambos sexos no se encontró un aumento

FIGURA 1. Comportamiento del contenido de sólidos totales para el extracto acuoso de $M$. charantia en el estudio de estabilidad. ${ }^{* *}$ diferencias significativas respecto al inicio del estudio $p<0.01$.

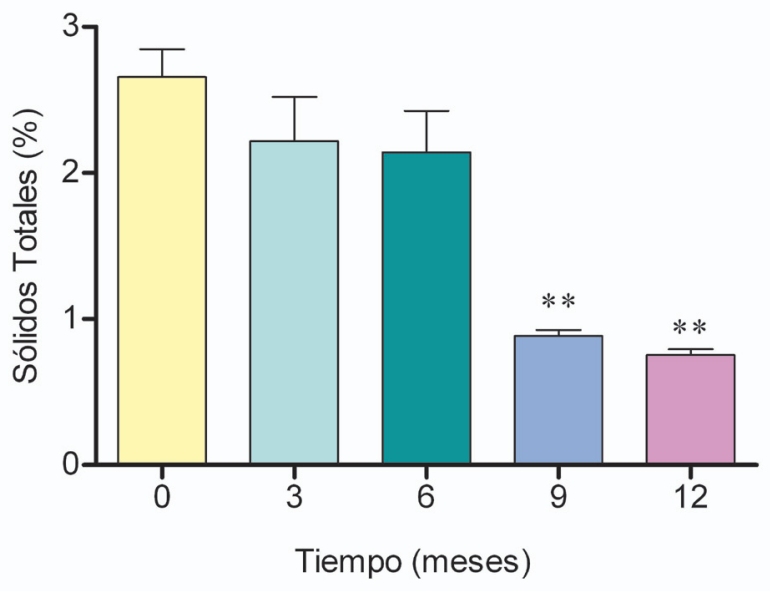

significativo $(p>0.05)$ en la frecuencia de eritrocitos policromáticos micronucleados (PCE-MN) de las dosis en estudio.

\section{Ensayo de aberraciones cromosómicas}

Se observó un equilibrio entre las aberraciones encontradas del tipo cromatídico y cromosómico encontrándose pocos intercambios, predominado los gaps. No se observó citotoxicidad y los valores de poliploidías de los tratamientos fueron muy similares al control no observándose diferencias significativas según $t$ Student (Tabla 3 ).

\section{Evaluación farmacológica}

Posterior a la inducción de hiperglicemia por carga de glucosa se observó un incremento de la glicemia en los animales de todos los grupos experimentales. En la Figura 2 se muestra el perfil de la glicemia en el tiempo. Se observó efecto hipoglicemiante en los grupos tratados con la dosis de $450 \mathrm{mg} / \mathrm{kg}$ de extractos de M. charantia, así como en el grupo control positivo administrado con Glibenclamida. Los animales tratados con el extracto a las dosis de 50 y $150 \mathrm{mg} / \mathrm{kg}$ mostraron un comportamiento muy similar al grupo control, observándose niveles elevados de glicemia durante todo el ensayo. La administración de estas dosis de extracto no modificaron las concentraciones séricas de glucosa.

\section{DISCUSIÓN}

Diversos estudios preclínicos y clínicos reportan el efecto hipoglicemiante de M. charantia. Se plantea que el principal mecanismo de acción de esta planta por el cual ejerce su efecto hipoglicemiante es por su acción antioxidante y protección de las células $\beta$ (Jun Yin et al. 2008).

La caracterización de los extractos obtenidos de esta planta cultivada en nuestro país, es indispensable para la futura obtención de un medicamento herbario con acción hipoglicemiante.

TABLA 2. Índice de Toxicidad (IT) y de Genotoxicidad (IG) de los grupos controles y tratados. MN: micronúcleos PCE: Eritrocitos policromáticos NCE: Eritrocitos normocromáticos. Los valores reflejan la media \pm desviación estándar. ${ }^{* *} p<0.01$ (Test de Dunnett).

\begin{tabular}{lcccc}
\hline \multirow{2}{*}{ Tratamiento } & \multicolumn{2}{c}{ IT (PCE/NCE) } & \multicolumn{2}{c}{ IG (PCE-MN) } \\
\cline { 2 - 5 } & \multicolumn{1}{c}{ Machos } & Hembras & Machos & Hembras \\
\hline Vehículo & $1.29 \pm 0.1$ & $1.33 \pm 0.07$ & $0.90 \pm 0.05$ & $0.86 \pm 0.16$ \\
M. charantia $112.5 \mathrm{mg} / \mathrm{kg}$ & $1.65 \pm 0.11$ & $1.63 \pm 0.08$ & $0.80 \pm 0.10$ & $0.84 \pm 0.20$ \\
M. charantia $225 \mathrm{mg} / \mathrm{kg}$ & $1.54 \pm 0.21$ & $1.55 \pm 0.18$ & $0.82 \pm 0.16$ & $0.84 \pm 0.11$ \\
M. charantia $450 \mathrm{mg} / \mathrm{kg}$ & $1.59 \pm 0.13$ & $1.61 \pm 0.29$ & $0.81 \pm 0.21$ & $0.83 \pm 0.18$ \\
Ciclofosfamida $40 \mathrm{mg} / \mathrm{kg}$ & $0.93 \pm 0.24$ & $0.86 \pm 0.13$ & $3.08 \pm 1.18^{* *}$ & $4.74 \pm 1.21^{\text {** }}$ \\
\hline
\end{tabular}

Rev. Bras. PI. Med., Campinas, v.16, n.4, p.782-788, 2014. 
TABLA 3. Resultados obtenidos en el ensayo de aberraciones cromosómicas del extracto hidroalcohólico de M. charantia.

\begin{tabular}{|c|c|c|c|c|c|c|}
\hline \multirow{3}{*}{$\begin{array}{l}\text { Concentración } \\
(\mathrm{mg} / \mathrm{ml})\end{array}$} & \multirow{3}{*}{$\begin{array}{l}\text { Metafases } \\
\text { analizadas }\end{array}$} & \multicolumn{5}{|c|}{ Aberraciones/200células } \\
\hline & & \multirow[t]{2}{*}{ Gaps } & \multicolumn{2}{|c|}{ Cromosómicas } & \multicolumn{2}{|c|}{ Cromatídicas } \\
\hline & & & Roturas & Intercambios & Roturas & Intercambios \\
\hline 0 & 200 & 1 & 1 & 0 & 0 & 0 \\
\hline 1 & 200 & 1 & 1 & 0 & 0 & 0 \\
\hline 2 & 200 & 2 & 1 & 0 & 1 & 0 \\
\hline 5 & 200 & 2 & 2 & 0 & 0 & 0 \\
\hline Mitomicina C & 200 & 7 & 8 & 11 & 9 & 14 \\
\hline
\end{tabular}
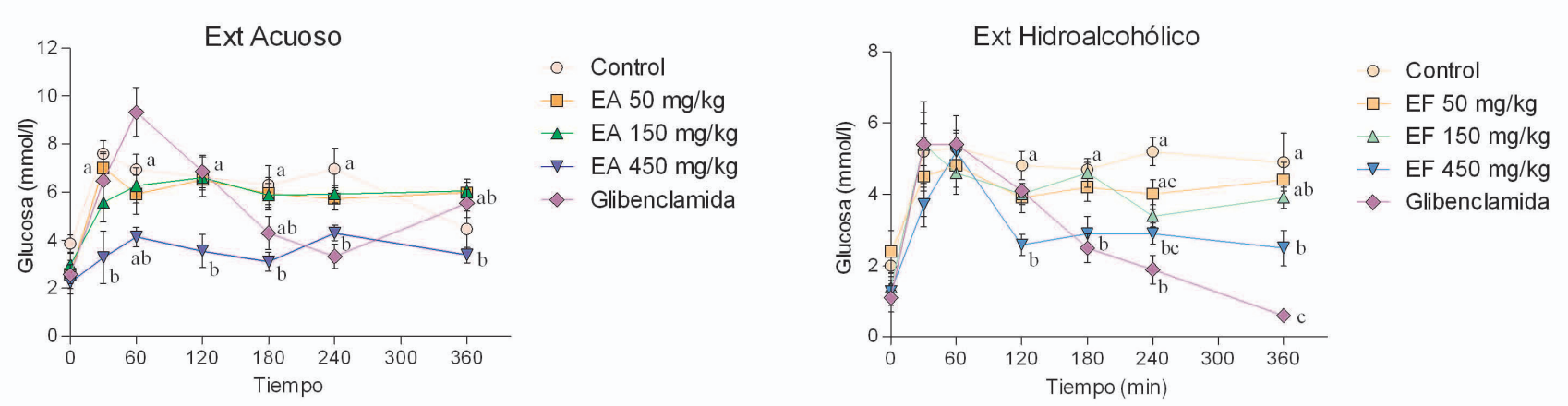

FIGURA 2. Comportamiento de los niveles de glucosa en animales hiperglicémicos tras tratamiento con extracto acuoso (EA) e hidroalcohólico (EF) de M. charantia. Los valores representan media \pm error estándar. Letras desiguales muestran diferencias significativas entre grupos $(p<0.05)$.

En el presente trabajo se aborda la obtención y caracterización de extractos acuoso e hidroalcohólico a partir de M. charantia.

El extracto acuoso fue obtenido mediante el método de reflujo, resultando estable por un período de 6 meses. La evaluación farmacológica mostró que la administración oral del extracto acuoso de $M$. charantia presenta actividad hipoglicemiante a la dosis de $450 \mathrm{mg} / \mathrm{kg}$ en ratas sanas con hiperglicemia temporal, impidiendo el incremento de los niveles de glicemia tras la carga de glucosa. Este efecto fue superior al observado para la Glibenclamida.

Por otra parte el extracto hidroalcohólico se obtuvo por repercolación y resultó estable por un período de 12 meses, manteniendo los índices de calidad durante este tiempo. Para este extracto se evaluó el potencial genotóxico mediante los ensayos de micronúcleos en médula ósea de ratón y aberraciones cromosómicas en linfocitos de sangre periférica, no mostrando genotoxicidad en el rango de dosis evaluado.

El extracto evaluado no indujo aberraciones cromosómicas en linfocitos de sangre periférica en las condiciones ensayadas ya que no se observó un aumento reproducible del número de células que presentan aberraciones cromosómicas, ni se observó un aumento de células poliploides que indican que el extracto es capaz de inhibir procesos mitóticos. En el ensayo in vivo de inducción de micronúcleos en médula ósea de ratón, para ambos sexos ninguna dosis causó toxicidad detectable en la médula ósea. Por otra parte el análisis estadístico del porciento de eritrocitos jóvenes micronucleados indica que no se encontraron diferencias estadísticamente significativas entre los sexos, las dosis empleadas, ni una relación dosis/respuesta lo que sugiere que este producto no es genotóxico.

La evaluación farmacológica del extracto hidroalcohólico mostró resultados similares al acuoso, observando efecto hipoglicemiante a la dosis de $450 \mathrm{mg} / \mathrm{kg}$ similar a la Glibenclamida.

La presencia de actividad hipoglicemiante en los extractos se corresponde con lo reportado por Khan et al (1993) quienes en ratas normales en ayunas hallaron una disminución de los niveles de glucosa tras el tratamiento con un extracto de $M$. charantia. Este efecto ha sido demostrado además en diferentes modelos animales (Miura et al. 2004; Zheng et al. 2005; Sathishsekar \& Subramanian, 2005).

Diversos trabajos tratan sobre la actividad hipoglicemiante de los frutos, el zumo y el extracto etanólico al $95 \%$ a dosis $3 \mathrm{~mL} / \mathrm{kg}$ por vía oral. En estos estudios, se responsabilizó de la actividad 
al polipéptido $\mathrm{P}$ (o V-insulina) que posee actividad hipoglicemiante en el hombre y en animales (Khanna et al. 1981).

Algunos componentes de $M$. charantia son estructuralmente similares a la insulina animal, y estudios preliminares han sugerido que disminuye la gluconeogénesis hepática e incrementa la síntesis de glucógeno hepático y la oxidación de la glucosa periférica en eritrocitos y adipositos. Un mecanismo propuesto para justificar la actividad hipoglicemiante se cree sea el aumento en la producción de las células $\beta$ del páncreas. Varios son los constituyentes de la M. charantia que se ha encontrado que poseen propiedades hipoglicemiantes; sin embargo el interés se ha centrado principalmente en el polipéptido $P$, anteriormente mencionado, y la combinación de dos glicósidos de esteroides conocidos como charantina (Basch \& Gabardi, 2003).

Por otra parte, ambos extractos fueron evaluados en ensayos de toxicidad aguda oral mostrando ligera toxicidad para el extracto hidroalcohólico dado por signos tóxicos y disminución del peso corporal en la primera semana del ensayo tras la administración aguda de $2000 \mathrm{mg} / \mathrm{kg}$ por vía oral, lo cual se debe principalmente a la presencia del etanol en el extracto. El extracto acuoso no mostró toxicidad significativa a la misma dosis. Ambos extractos, acuoso e hidroalcohólico, se clasificaron en categoría $5\left(\mathrm{DL}_{50}>2000 \mathrm{mg} / \mathrm{kg}\right)$ de la OECD según el ensayo de toxicidad aguda oral y resultaron no irritantes en ojo y piel de conejo (Lagarto et al. 2008).

En conclusión, se establecieron las especificaciones de calidad de extractos acuoso e hidroalcohólico a partir del follaje de M. charantia y mostraron buena estabilidad. Su potencial genotóxico fue evaluado en el extracto hidroalcohólico el cual no mostró toxicidad en los dos ensayos evaluados. La evaluación farmacológica evidenció efecto hipoglicemiante en ratas con hiperglicemia temporal a la dosis de $450 \mathrm{mg} / \mathrm{kg}$. Ambos productos constituyen buenos candidatos para desarrollar productos finales destinados al tratamiento de la diabetes, mostrando efecto farmacológico hipoglicemiante y baja toxicidad.

\section{REFERENCIAS}

AHMED, I.; ADEGHATE, E.; CUMMINGS, E.; SHARMA, A.K.; SINGH, J. Beneficial effects and mechanism of action of Momordica charantia juice in the treatment of streptozotocin-induced diabetes mellitus in rat. Mollecular Cell Biochemestry, v.261, n.1-2, p.63-70, 2004.

BASCH, E.; GABARDI, S.; ULBRICHT. Bitter melon (Momordica charantia): a review of efficacy and safety. American Journal of Health-System Pharmacists, v.60, p.356-9, 2003.

CERIELLO, A. Postpandrial hyperglycemia and diabetes complications: is it time to treat? Diabetes, v.54, p.1-7, 2005.

CHATURVEDI, P.; GEORGE, S.; MILINGANYO, M.; TRIPATHI, Y.B. Effect of Momordica charantia on lipid profile and oral glucose tolerance in diabetic rats. Phytotherapy Research, v.18, n.11, p.954-6, 2004.

CHEN, Q.; CHAN, L.L.; LI, E.T. Bitter melon (Momordica charantia) reduces adiposity lowers serum insulin and normalizes glucose tolerance in rats fed a high fat diet. Journal of. Nutrition, v.133, p.1088-93, 2003.

CYTED. Programa Iberoamericano de Ciencia y Tecnología para el Desarrollo. Manual de Técnicas de Investigación. 1995.

HARTMANN, A.; AGURELL, E.; BEEVERS, C; BRENDLER-SCHWAAB, S.; BURLINSON, B.; CLAY, P.; COLLINS, A.; SMITH, A.; SPEIT, G.; THYBAUD, V.; TICE, R.R. Recommendations for conducting the in vivo alkaline Comet assay. Mutagenesis, v.18, n.1, p. 45-51, 2003.

HAYASHI, M.; TICE, R.R.; MCGREGOR, J.T.;ANDERSON, D.; BLAKEY, D.H.; KIRSH-VOLDERS, M.; OLESON, F.B. Jr; PACCHIEROTTI, F.; ROMAGNA, F.; SHIMADA, $H$. Et al. In vivo rodent erythrocyte micronucleus assay. Mutation Research, v.312, n.3, p.293-304, 1994.

YIN, J.; ZHANG, H.; YE, J. Traditional Chinese Medicine in Treatment of Metabolic Syndrome. Endocrine, Metabolic and Immune Disorders Drug Targets, v.8, n.2, p.99-111, 2008.

KHAN, A.L.; MAMUN, A.K.A., MOSHUZZAMAN, M.I.R.; NAHAR, M.; ALAM, N.; ROKEYA, M.N. Studies on hypoglycemic effects of fruit pulp seed and whole plant of Momordica charantia on normal and diabetic model rats. Planta Medica, v.59, p.408-12, 1993.

KHANNA, P.; JAIN, S.C.; PANAGARIYA, A.; DIXIT, V.P. Hypoglicemic Activity of polypeptide $-P$ from a plant source. Journal of Natural Products, v.44, n.6, p.64855, 1981.

LAGARTO, A.; COURET, M.; GUERRA, I.; LÓPEZ, R. Toxicidad aguda oral y ensayos de irritación de extractos acuoso e hidroalcohólico de Momordica charantia Lin. Revista Cubana de Plantas Medicinales, v.13, n.3, 2008. Disponible en www.sld.cu. Acceso en abril.2011.

MINISTERIO DE SALUD PÚBLICA. NRSP - 309. Medicamentos de origen vegetal. 1992.

MINISTERIO DE SALUD PÚBLICA. NRSP - 311. Medicamentos de origen vegetal. 1992.

MIURA, T.; ITOH, Y., IWAMOTO, N.; KATO, M.; ISHIDA, T. Suppressive activity of the fruit of Momordica charantia with exercise on blood glucose in type 2 diabetic mice. Biological and Pharmaceutical Bulletin, v.27, n.2, p.248-50, 2004.

MIURA, T.; ITOH, Y.; IWAMOTO, N.; KAWAI, M.; PARK, S.R.; SUZUKI, I. Hypoglycemic activity of the fruit of the Momordica charantia in type 2 diabetic mice. Journal of Nutritional Sciences and Vitaminology, v.47, n.5, p. 340-4, 2001.

ROIG Y MESA, J.T. Plantas Medicinales, Aromáticas - Venenosas de Cuba. La Habana: Instituto del Libro Ciencia y Técnica, 1974. p.318 - 20.

SATHISHSEKAR, D.; SUBRAMANIAN, S. Beneficial effects of Momordica charantia seeds in the treatment of STZ- 
induced diabetes in experimental rats. Biological and Pharmaceutical Bulletin, v.28, n.6, p.978-83, 2005.

SCHMID, W. The micronucleus test for cytogenetic analysis. Chemical Mutagens, v.4, p.31, 1976.

TICE, R.R. The single cell gel/comet assay: a microgel electrophoretic technique for the detection of DNA damage and repair individual cells. In: PHILLIPS, D.H.; VENITT, S. (Eds.), Environmental Mutagenesis. Oxford: Bios Scientific Publishers LTD, 1995. p. 315-
39.

WAGNER, H.; BLADT, S. Plant Drug Analysis. A thin Layer Chromatography Atlas. 2.ed. Berlin: Springer, 2001. 368p.

ZHENG, Z.X.; TENG, J.Y.; LIU, J.Y.; QIU, J.H.; OUYANG, $\mathrm{H}$.; XUE, C. The hypoglycemic effects of crude polysaccharides extract from Momordica charantia in mice. Wei Sheng Yan Jiu (Journal of Hygiene Research), v.34, n.3, p.361-3, 2005. 